\title{
Clinical and economic burden of procedural sedation-related adverse events and their outcomes: analysis from five countries
}

This article was published in the following Dove Press journal:

Therapeutics and Clinical Risk Management

\author{
Rhodri Saunders' \\ Jason A Davis' \\ Peter Kranke ${ }^{2}$ \\ Rachel Weissbrod ${ }^{3}$ \\ David K Whitaker ${ }^{4}$ \\ Jenifer R Lightdale ${ }^{5}$ \\ 'Coreva Scientific GmbH \& Co KG, \\ Freiburg, Germany; ${ }^{2}$ Department \\ of Anaesthesia and Critical Care, \\ University of Würzburg, Würzburg, \\ Germany; ${ }^{3}$ Medical Affairs, Medtronic, \\ Jerusalem, Israel; ${ }^{4}$ Department of \\ Anaesthesia, Manchester Royal \\ Infirmary, Manchester, UK; ${ }^{5}$ Pediatric \\ Gastroenterology, Hepatology \\ and Nutrition, University of \\ Massachusetts, Worcester, MA, USA
}

Correspondence: Rhodri Saunders Coreva Scientific GmbH \& Co KG, Kaiser-Joseph-Straße 198-200, Freiburg 79098, Germany

Tel +4976176999422

Fax +49 76I 76999421

Email rhodri@coreva-scientific.com
Background: Studies have reported on the incidence of sedation-related adverse events (AEs), but little is known about their impact on health care costs and resource use.

Methods: Health care providers and payers in five countries were recruited for an online survey by independent administrators to ensure that investigators and respondents were blinded to each other. Surveys were conducted in the local language and began with a "screener" to ensure that respondents had relevant expertise and experience. Responses were analyzed using Excel and R, with the Dixon's $Q$ statistic used to identify and remove outliers. Global and country-specific average treatment patterns were calculated via bootstrapping; costs were mean values. The sum product of costs and intervention probability gave a cost per AE.

Results: Responses were received from 101 providers and 26 payers, the majority having $>5$ years of experience. At a minimum, the respondents performed a total of 3,430 procedural sedations per month. All AEs detailed occurred in clinical practice in the last year and were reported to cause procedural delays and cancellations in some patients. Standard procedural sedation costs ranged from $€ 74$ (Germany) to $\$ 2,300$ (US). Respondents estimated that AEs would increase costs by between $16 \%$ (Italy) and 179\% (US). Hypotension was reported as the most commonly observed AE with an associated global mean cost (interquartile range) of \$43 (\$27-\$68). Other frequent AEs, including mild hypotension, bradycardia, tachycardia, mild oxygen desaturation, hypertension, and brief apnea, were estimated to increase health care spending on procedural sedation by $\$ 2.2$ billion annually in the US.

Conclusion: All sedation-related AEs can increase health care costs and result in substantial delays or cancellations of subsequent procedures. The prevention of even minor AEs during procedural sedation may be crucial to ensuring its value as a health care service.

Keywords: costs, complications, moderate sedation, questionnaire, survey, health care payers

\section{Introduction}

Sedation and anesthesia are an important means of providing patient comfort, safety, and clinical stability during invasive and/or uncomfortable medical procedures. Use of procedural sedation is considered to be on the rise. For example, a recent meta-analysis reported a steady increase in rates of procedural sedation for gastrointestinal procedures from around 10\% in 2000 to $30 \%$ in 2010 in the US. ${ }^{1}$ A separate trend analysis indicated that of nonoperating room procedures, $69.7 \%$ in 2010 and $73.3 \%$ in 2014 used sedation. ${ }^{2}$ The use of procedural sedation is expected to continue to grow, in part due to advances in minimally invasive operative techniques and evidence that its use improves patient outcomes. ${ }^{3}$ Concomitantly, patients' expectations regarding comfort, safety and clinical outcomes have increased, making sedation quality an important area of investigation. ${ }^{4}$ 
Procedural sedation carries inherent patient risks and requires careful monitoring of patients' vital signs. Both US (American Society of Anesthesiologists) and European (European Board of Anaesthesiology and European Society of Anaesthesiology) recommendations in this regard are broadly in agreement, requiring minimum measures of oxygenation (pulse oximetry), circulation (non-invasive blood pressure), and ventilation (capnography). ${ }^{5,6}$ Despite best monitoring practices, however, adverse events (AEs) related to sedation still occur, with hypotension and hypoxemia among the most commonly reported. ${ }^{7}$ These AEs have been documented across all studies of sedation, although the definitions used for each have been heterogeneous and rates have varied by study type. For example, prospective randomized controlled trials $^{8-11}$ have generally reported higher rates of sedationrelated AEs compared with retrospective studies. ${ }^{12-14}$

Given the potential for sedation-related AEs, providers around the world have learned to choose sedation plans that consider both safety and costs. Optimization of sedation planning requires knowledge of current clinical practice in sedation and monitoring, how AEs are perceived and treated and potential costs should an AE occur. Many clinical practice reviews focus on discussions of ideal agents, ${ }^{15}$ risk stratification of subpopulations, ${ }^{16,17}$ and/or settings. ${ }^{12,16}$ Although informative, these focused studies have provided neither a generalized overview of sedation practice in a global context nor an informed perspective on AE management or costs. As such, the precise data required to inform value-based decisions around sedation practices have not been available.

Our study was designed to address this data gap and provide a global context that could be useful to providers and payers involved in the administration of procedural sedation. We sought to estimate the economic burden of sedation-related AEs across multiple countries and medical practices. Our aim was to understand current clinical sedation practice used by different providers and interventions used to treat AEs, as defined by the World Society for Intravenous Anesthesia (SIVA) task force on procedural sedation. ${ }^{18}$ We also obtained survey data from local payers on the cost of interventions and resource use, and our analyses provide a first look at global practice in management of AEs, finding both consistencies in practice and variations in costs associated with sedation-related AEs.

\section{Materials and methods}

The aim of our study was to provide an estimate of the cost of AEs associated with sedation from the perspective of the hospital payer. Two surveys were prepared: one for providers qualified to oversee procedural sedation and one for payers (allowing those in clinics, hospitals, or medical offices or state health insurers to participate). Both surveys were designed and reviewed by the study authors and administered by a third party (GfK Switzerland), thereby blinding the authors to participants and vice versa. As this was a pilot study, no power calculations were performed to determine a sample size for number of respondents required per country; 20 providers and five payers were deemed feasible quotas for each target country. Surveys were designed in English (administered in the UK and US) and performed in local language in France, Germany and Italy.

Our study was designed as a general survey of practice patterns and costing data and involved surveying an array of individuals in different roles across different institutions worldwide about their knowledge, attitudes and beliefs. No patient-level data were included, and the survey was not subject to an institutional review process. Survey respondents provided informed consent for participation in the research and its publication through direct enquiry by reading and agreeing to an introductory statement explaining the purpose and use of the data prior to completing the survey items.

Each survey consisted of two sections: a screener and a questionnaire. The screener questions ensured that only respondents with relevant expertise and experience completed the questionnaire. Screener questions included items such as time employed in health care and number of procedural sedations administered per month.

The provider questionnaire collected data on sedation agents, patient monitoring practices, relative incidence of AEs encountered, common management strategies for AEs, and outcomes following AEs (Supplementary materials). For treatment and outcomes of AEs, respondents were provided an AE and its SIVA definition and were asked to provide whole numbers of patients who would receive a corresponding treatment (including no treatment) from a list of SIVA-defined interventions. In the interest of reducing the time burden to complete the survey, only a subset of AEs was presented to each respondent. These AEs queried were random unless a respondent indicated that they had experience with a rare $\mathrm{AE}$, in which case the rare event was preferentially queried.

The payer questionnaire collected data on the direct and comprehensive (or "fully loaded" costs, inclusive of administration, management, quality, legal and transport services) costs of procedural sedation (including all SIVAdefined interventions) and health care resources (including provider time). Respondents were also asked to provide the 
overall cost of an uncomplicated procedural sedation and then to estimate the percentage increase in costs resulting from procedural sedation-related complications. Costs provided were those determined by the individual payer respondent; no specific costing methodology was enforced on the payer by the survey. Owing to high degrees of variability and subjectivity, particularly regarding potential legal obligations, only the direct costs of interventions were used to calculate costs of AEs in this study. These results were compared to the overall ("fully loaded") costs reported by payers for procedures with sedation-related complications. Values were entered in the local currencies, where " 0 " indicated no direct costs to the institution.

Data were first assessed in Microsoft Excel, with subsequent cleaning and analysis performed using Microsoft Excel and R. Analysis of AEs and treatment patterns adopted an "identify and replace" strategy for response outliers. Outliers (at the $95 \%$ confidence level or $p$-value $<0.05$, Dixon's $Q$ test) were replaced with the global mean for that combination of $\mathrm{AE}$ and treatment response. Average treatment patterns were calculated via the weighted mean treatment pattern per AE per country. Higher weight was given to respondents who reported more experience of the $\mathrm{AE}$, and lower weight was given to respondents with more outlier responses. Global median patterns and interquartile ranges (IQRs) were derived from 10,000 bootstrap replicates. Statistical inference testing was performed using nonparametric methods. To assess differences among multiple groups, the Kruskal-Wallis test was applied at $p$-value $\leq 0.05$ for significance of results.

Payer respondents had the option to report no cost or cost unknown, resulting in anywhere from two to five responses for cost items in each country. Since these counts were low, bootstrapping was not performed to estimate uncertainties. Instead, a simple mean of the provided values was calculated in the local currency.

Analysis of costs was performed with the same outlier identification strategy. Costs were obtained in February 2017 and March 2017 and were assumed to represent costs for the 2016-2017 period. The overall direct cost of each AE was calculated in two parts, the intervention cost and the personnel cost. As the first step in calculating costs of sedation-related AEs, provider data were analyzed to determine the frequencies at which various interventions were used to address the presented AEs. The intervention frequencies were multiplied by the payer-reported costs of individual interventions to estimate the intervention cost of each AE. Time to treat the AE was multiplied by the number of providers present and the mean reported wage to determine the personnel burden.
Combining costs and treatment patterns, the mean cost per AE per country was calculated, and a "global mean cost" was determined by first converting each country's mean cost to 2016 USD using the average annual exchange rate from the Internal Revenue Service of the US. The average of the five countries was taken as the global mean cost.

\section{Results \\ Demographics}

Respondents to the provider survey comprised multiple medical practices and settings, with most having $>5$ years of experience (Table 1). At a minimum, the 101 providers sampled represent 3,430 procedural sedations per month: France (700), Germany (730), Italy (700), UK (550) and US (750).

There were 26 respondents to the payer survey, with six respondents in France and five each in the other countries. The majority (23) were hospital-employed payers, with two employed by a clinic and one in a national health service. Most common job titles were department head (six), procurement (five), and manager (five). All included responsibility for purchasing, pricing, and/or resources.

\section{Expected costs of procedural sedation}

Prior to calculation of costs of complications in procedural sedation, the payer data were used to provide an overview of current estimates of overall sedation costs. The payer responses revealed wide differences in the costs of procedural sedation and the impact of complications (Table 2) with mean costs ranging from EUR 74 in Germany to USD 2,300 in the US. Complications resulted in cost increases of 12\% (Italy) to $78 \%$ (Germany) with a global mean of $47 \%$.

\section{Current clinical practice}

A snapshot of provider responses for current clinical practices found most were more likely to follow local hospital guidelines than national and society guidelines (Table 1). The use of sedation agents was broadly similar across settings, although use of fentanyl and ketamine showed differences by geography $(p<0.01)$. Among respondents, the most common patient monitoring modality used was pulse oximetry, which was also most likely to be considered standard of care and to be used to guide clinical management $(p<0.05)$. The least used monitoring modality among those analyzed was capnography.

\section{AEs and their treatment}

All AEs surveyed were reported to have occurred in clinical practice in the last year by at least one respondent per country. 
Table I Demographics and select procedural sedation parameters of survey respondents

\begin{tabular}{|c|c|c|c|c|c|c|}
\hline Item & Global & France & Germany & Italy & UK & US \\
\hline$n$ & 101 (100.0\%) & $20(100.0 \%)$ & $20(100.0 \%)$ & 20 (100.0\%) & 20 (100.0\%) & $21(100.0 \%)$ \\
\hline \multicolumn{7}{|l|}{ Medical practice } \\
\hline Sedation nurse & 47 (46.5\%) & $9(45.0 \%)$ & II (55.0\%) & $7(35.0 \%)$ & $10(50.0 \%)$ & $10(47.6 \%)$ \\
\hline Anesthesiologist & 31 (30.7\%) & $10(50.0 \%)$ & $5(25.0 \%)$ & $5(25.0 \%)$ & $5(25.0 \%)$ & $6(28.6 \%)$ \\
\hline Gastroenterologist & $16(15.8 \%)$ & $0(0.0 \%)$ & $3(15.0 \%)$ & $8(40.0 \%)$ & $3(15.0 \%)$ & $2(9.5 \%)$ \\
\hline Cardiologist & $3(3.0 \%)$ & $0(0.0 \%)$ & $0(0.0 \%)$ & $0(0.0 \%)$ & I (5.0\%) & $2(9.5 \%)$ \\
\hline Critical care specialist & $3(3.0 \%)$ & I (5.0\%) & I (5.0\%) & $0(0.0 \%)$ & I (5.0\%) & $0(0.0 \%)$ \\
\hline Plastic surgeon & I (I.0\%) & $0(0.0 \%)$ & $0(0.0 \%)$ & $0(0.0 \%)$ & $0(0.0 \%)$ & I (4.8\%) \\
\hline \multicolumn{7}{|l|}{ Associated setting } \\
\hline General hospital & $52(51.5 \%)$ & $9(45.0 \%)$ & 14 (70.0\%) & $7(35.0 \%)$ & $12(60.0 \%)$ & $10(47.6 \%)$ \\
\hline University hospital & $36(35.6 \%)$ & $9(45.0 \%)$ & $5(25.0 \%)$ & $8(40.0 \%)$ & $9(45.0 \%)$ & $5(23.8 \%)$ \\
\hline Private hospital & $16(15.8 \%)$ & $0(0.0 \%)$ & I (5.0\%) & $6(30.0 \%)$ & $3(15.0 \%)$ & $6(28.6 \%)$ \\
\hline Private clinic & II (I0.9\%) & $3(15.0 \%)$ & $0(0.0 \%)$ & $3(15.0 \%)$ & $0(0.0 \%)$ & $5(23.8 \%)$ \\
\hline Physician's office & $5(5.0 \%)$ & I (5.0\%) & $0(0.0 \%)$ & I (5.0\%) & $0(0.0 \%)$ & $3(14.3 \%)$ \\
\hline Hospital outpatient clinic & $4(4.0 \%)$ & $0(0.0 \%)$ & $0(0.0 \%)$ & $0(0.0 \%)$ & $0(0.0 \%)$ & $4(19.0 \%)$ \\
\hline Ambulatory surgical center & $2(2.0 \%)$ & $0(0.0 \%)$ & $0(0.0 \%)$ & $0(0.0 \%)$ & $0(0.0 \%)$ & $2(9.5 \%)$ \\
\hline \multicolumn{7}{|l|}{ Years of experience } \\
\hline $5+$ & $8(7.9 \%)$ & $3(15.0 \%)$ & $0(0.0 \%)$ & I (5.0\%) & $3(15.0 \%)$ & I (4.8\%) \\
\hline $2-5$ & $93(92.1 \%)$ & $17(85.0 \%)$ & $20(100.0 \%)$ & $19(95.0 \%)$ & $17(85.0 \%)$ & $20(95.2 \%)$ \\
\hline \multicolumn{7}{|l|}{ Procedures per month } \\
\hline $20-50$ & $54(53.5 \%)$ & $10(50.0 \%)$ & $9(45.0 \%)$ & $10(50.0 \%)$ & $\mathrm{I} 5$ (75.0\%) & $10(47.6 \%)$ \\
\hline $50+$ & 47 (46.5\%) & $10(50.0 \%)$ & II (55.0\%) & $10(50.0 \%)$ & $5(25.0 \%)$ & II (52.4\%) \\
\hline \multicolumn{7}{|l|}{ Sedation agents used } \\
\hline Midazolam & $93(92.1 \%)$ & $16(80.0 \%)$ & 17 (85.0\%) & $20(100.0 \%)$ & $20(100.0 \%)$ & $20(95.2 \%)$ \\
\hline Propofol & $90(89.1 \%)$ & $19(95.0 \%)$ & $20(100.0 \%)$ & $18(90.0 \%)$ & $15(75.0 \%)$ & $18(85.7 \%)$ \\
\hline Fentanyl & 75 (74.3\%) & $9(45.0 \%)$ & $16(80.0 \%)$ & $18(90.0 \%)$ & $18(90.0 \%)$ & $14(66.7 \%)$ \\
\hline Ketamine & $56(55.4 \%)$ & $18(90.0 \%)$ & $10(50.0 \%)$ & 7 (35.0\%) & $9(45.0 \%)$ & $12(57.1 \%)$ \\
\hline Meperidine & 15 (14.9\%) & I (5.0\%) & $2(10.0 \%)$ & $6(30.0 \%)$ & $4(20.0 \%)$ & $2(9.5 \%)$ \\
\hline \multicolumn{7}{|l|}{ Monitoring methods used } \\
\hline Pulse oximetry & 99 (98.0\%) & $19(95.0 \%)$ & $20(100.0 \%)$ & $20(100.0 \%)$ & $19(95.0 \%)$ & $21(100.0 \%)$ \\
\hline Heart rate & 97 (96.0\%) & $18(90.0 \%)$ & $20(100.0 \%)$ & $19(95.0 \%)$ & $19(95.0 \%)$ & $21(100.0 \%)$ \\
\hline Blood pressure & $95(94.1 \%)$ & $18(90.0 \%)$ & $20(100.0 \%)$ & $16(80.0 \%)$ & $20(100.0 \%)$ & $21(100.0 \%)$ \\
\hline ECG & 91 (90.1\%) & 15 (75.0\%) & $20(100.0 \%)$ & $18(90.0 \%)$ & $17(85.0 \%)$ & $21(100.0 \%)$ \\
\hline Respiratory rate & $82(81.2 \%)$ & $16(80.0 \%)$ & 15 (75.0\%) & $12(60.0 \%)$ & $18(90.0 \%)$ & $21(100.0 \%)$ \\
\hline Capnography & 62 (6I.4\%) & II (55.0\%) & $14(70.0 \%)$ & $8(40.0 \%)$ & $13(65.0 \%)$ & $16(76.2 \%)$ \\
\hline \multicolumn{7}{|l|}{ Source of guidelines used } \\
\hline Local & $70(69.3 \%)$ & $12(60.0 \%)$ & $17(85.0 \%)$ & $10(50.0 \%)$ & $14(70.0 \%)$ & $17(81.0 \%)$ \\
\hline National & $57(56.4 \%)$ & $12(60.0 \%)$ & II (55.0\%) & $10(50.0 \%)$ & II (55.0\%) & $13(61.9 \%)$ \\
\hline Professional society & $43(42.6 \%)$ & $10(50.0 \%)$ & $13(65.0 \%)$ & $4(20.0 \%)$ & $4(20.0 \%)$ & $12(57.1 \%)$ \\
\hline International & $17(16.8 \%)$ & $5(25.0 \%)$ & $3(15.0 \%)$ & $6(30.0 \%)$ & $2(10.0 \%)$ & I (4.8\%) \\
\hline
\end{tabular}

Note: Numbers in parentheses indicate percentages globally or by country.

Abbreviation: ECG, electrocardiography.

Global median treatment patterns for AEs are presented in Table 3, ordered by relative global incidence as reported by respondents from most frequent (hypotension) to least (seizure). Data were relatively consistent across countries; a test of $\mathrm{AE} /$ treatment pattern combinations across countries found that $89.4 \%$ of these were not significantly different at the $95 \%$ confidence level ( $p$-value $>0.05$ ). Of the remaining $10.6 \%$ with significant differences, the most inter-country

Table 2 Reported procedural sedation costs

\begin{tabular}{llllll}
\hline Type of procedural sedation & France (EUR) & Germany (EUR) & Italy (EUR) & UK (GBP) & US (USD) \\
\hline Cost of uncomplicated procedure & $550(300-1,000)$ & $74(0-150)$ & $263(0-800)$ & $570(0-1,500)$ & $2,030(I 50-5,000)$ \\
Cost with sedation-related complication & $857(390-1,500)$ & $132(0-300)$ & $294(0-880)$ & $758(0-2,250)$ & $3,200(600-6,250)$ \\
Estimated costs of complications & $258(90-500)$ & $57(0-150)$ & $31(0-80)$ & $188(0-750)$ & $1,170(400-2,500)$ \\
\hline
\end{tabular}

Notes: Payers were asked to provide costs for uncomplicated sedation procedures ("fully loaded" costs, inclusive of administration, management, quality, and legal and transport services) and individual costs of procedures with complications. The cost differences due to complications were calculated, and mean values and ranges were determined. All values are mean values with range in parentheses. 


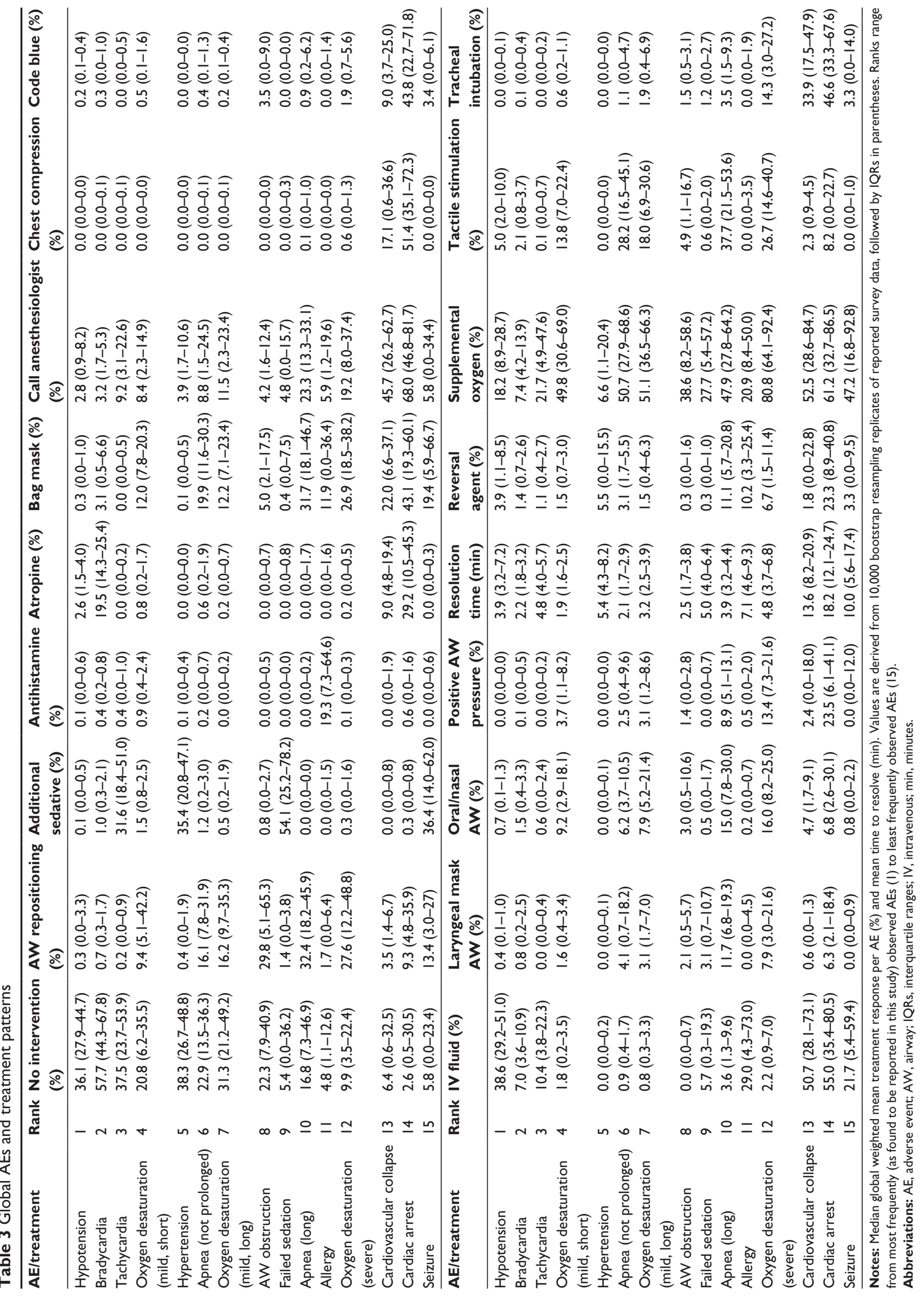


Table 4 Costs of AEs

\begin{tabular}{|c|c|c|c|c|c|c|c|}
\hline AE & Rank & France (EUR) & Germany (EUR) & Italy (EUR) & UK (GBP) & US (USD) & World $^{\mathrm{a}}$ (USD) \\
\hline Hypotension & 1 & $23(18-28)$ & $6(4-8)$ & $9(6-13)$ & $14(10-18)$ & $210(154-269)$ & $43(27-68)$ \\
\hline Bradycardia & 2 & II (7-I5) & $5(3-7)$ & $7(5-10)$ & $14(9-19)$ & $63(40-87)$ & $25(13-45)$ \\
\hline Tachycardia & 3 & $43(3 I-54)$ & $5(3-6)$ & $7(4-I I)$ & $68(43-86)$ & $198(128-285)$ & $53(25-101)$ \\
\hline Oxygen desaturation (mild, short) & 4 & $16(|I-2|)$ & $10(7-15)$ & $10(7-14)$ & $20(I I-28)$ & $436(319-544)$ & $53(27-98)$ \\
\hline Hypertension & 5 & $24(15-30)$ & $6(5-7)$ & $9(5-12)$ & $37(26-49)$ & $114(70-162)$ & $46(24-74)$ \\
\hline Apnea (not prolonged) & 6 & $24(18-32)$ & $73(26-113)$ & $18(1 \mid-24)$ & $16(9-24)$ & $340(237-434)$ & $70(36-128)$ \\
\hline Oxygen desaturation (mild, long) & 7 & $29(20-38)$ & II (5-I5) & II (7-15) & $20(12-28)$ & $367(28 I-459)$ & $57(3 \mid-108)$ \\
\hline AW obstruction & 8 & $20(9-30)$ & $19(13-28)$ & $15(7-23)$ & $17(8-26)$ & $196(54-29 \mid)$ & $57(7-134)$ \\
\hline Failed sedation & 9 & $43(15-56)$ & $22(|2-3|)$ & $15(12-18)$ & $32(15-49)$ & $183(7|-3| 0)$ & $80(30-142)$ \\
\hline Apnea (long) & 10 & $46(34-57)$ & $92(67-119)$ & $16(9-25)$ & $38(23-54)$ & $372(263-493)$ & $110(59-205)$ \\
\hline Allergy & 11 & $8(I-13)$ & $12(8-16)$ & $16(8-24)$ & $63(32-92)$ & 479 (209-707) & $48(1 \mathrm{I}-148)$ \\
\hline Oxygen desaturation (severe) & 12 & $55(4 I-70)$ & $66(37-88)$ & $35(24-45)$ & $39(22-55)$ & $501(379-617)$ & $130(68-223)$ \\
\hline Cardiovascular collapse & 13 & $46(3 I-62)$ & $122(83-163)$ & $19(7-28)$ & $180(|3|-233)$ & $\mathrm{I}, 986(\mathrm{I}, 456-2,5 \mathrm{I})$ & $217(90-447)$ \\
\hline Cardiac arrest & 14 & $70(40-102)$ & $188(|4|-234)$ & $66(48-84)$ & $597(44 I-823)$ & $3,597(3,108-4,055)$ & $575(3|2-92|)$ \\
\hline Seizure & 15 & $24(10-39)$ & $75(48-109)$ & $26(|2-4|)$ & $78(26-122)$ & $808(584-1,015)$ & $116(29-270)$ \\
\hline
\end{tabular}

Notes: Median costs (direct costs, not fully-loaded) were calculated from average treatment patterns by country or globally (from 10,000 bootstrap replicates) multiplied by mean costs per AE. Values for AE costs are followed by IQRs in parentheses. ${ }^{\text {FFor }}$ global medians (mean values for overall reported procedural sedation costs), costs were converted from each corresponding currency to USD using the average annual currency conversion rate. Ranks range from most frequently (as found to be reported in this study) observed AEs (I) to least frequently observed AEs (I5).

Abbreviations: $A E$, adverse event; $A W$, airway; IQR, interquartile range.

variability occurred in reports of rates of nonintervention in Italy for prolonged apnea and varied greatly from responses by providers in Germany, the UK, and the US.

Time required to treat AEs was consistently found to be $>1$ minute, even for mild events. Variations occurred across countries, however, with US-based providers more likely to intervene for mild AEs compared to their European counterparts. Invasive interventions (eg, endotracheal intubation for airway [AW] support) were reported more likely to occur with serious AEs, such as cardiovascular collapse.

\section{Costs of AEs}

Payers reported all treatment responses (except "none") had an associated cost. In contrast to the range of procedural sedation costs and variation in the impact of AEs on these costs as provided by payers (Table 2), the costs of AEs per procedure were found to be relatively consistent among countries (Table 4). The global median costs for cardiac arrest (\$575) and cardiovascular collapse (\$217) were the most expensive, while hypotension (\$43) and hypertension (\$46) were the least expensive (2016 USD). In general, unit costs were highest in the US and lowest in Italy. There was consistency in costs across European countries, and these appeared to differ from those of the US.

\section{$\mathrm{AE}$ outcomes and impact of potential cost}

The global median outcome expectations for each AE are presented in Table 5. Cardiac arrest and cardiovascular collapse were reported as the most serious AEs and led to the highest proportions of procedures delayed or terminated, hospital stays, and mortality. When compared to cardiovascular collapse, seizures resulted in a similar proportion of procedures delayed ( $47.6 \%$ seizure versus $49.1 \%$ cardiovascular collapse) and more procedures terminated ( $71.2 \%$ versus $54.1 \%)$, but a considerably lower mortality rate $(0.2 \%$ versus $2.4 \%)$.

All AEs were associated with the potential for early termination, delays in subsequent scheduled procedures, and inpatient stays. Although incidence of these outcomes could not be accurately estimated to allow for overall quantification of burden, costs and resource use associated with these outcomes were found to be substantial. The cost of canceling a procedure, for example, ranged from $€ 183$ in Germany to $€ 2,780$ in France. Early termination of a procedure was also found to be expensive in certain settings (eg, $\$ 2,320$ in the US).

\section{Per-procedure cost impact}

Using available absolute incidence data for the most commonly reported AEs (from Germany ${ }^{8,9,19}$ and the US ${ }^{11,20}$ ), mean (IQR) increase in cost for a complicated procedural sedation was calculated to be $€ 9$ (5-13) in Germany and $\$ 473$ (339-579) in the US. These data correspond to percentage increases over uncomplicated procedures of $12.7 \%$ and $23.3 \%$, respectively.

\section{Discussion}

We performed the first comparison of SIVA-defined AEs and their associated costs across multiple providers in different 
Table 5 Global AE outcomes

\begin{tabular}{|c|c|c|c|c|c|c|c|}
\hline AE/outcome & Rank & $\begin{array}{l}\text { Procedures } \\
\text { delayed (\%) }\end{array}$ & $\begin{array}{l}\text { Procedures } \\
\text { terminated (\%) }\end{array}$ & $\begin{array}{l}\text { Unplanned } \\
\text { inpatient } \\
\text { stays (\%) }\end{array}$ & $\begin{array}{l}\text { Length of } \\
\text { hospital stay } \\
\text { (days) }\end{array}$ & $\begin{array}{l}\text { Permanent } \\
\text { neurological } \\
\text { defect }(\%)\end{array}$ & Death (\%) \\
\hline Hypotension & 1 & $4.0(1.9-7.2)$ & $4.0(2.3-8.5)$ & $3.6(1.9-7.8)$ & $1.3(1 . I-1.7)$ & $0.5(0.2-0.9)$ & $0.2(0.1-0.4)$ \\
\hline Bradycardia & 2 & $2.4(1.3-5.1)$ & $2.8(1.4-5.5)$ & $2.6(1.4-5.6)$ & $1.2(1.1-1.9)$ & $0.2(0.0-0.6)$ & $0.1(0.0-0.3)$ \\
\hline Tachycardia & 3 & $2.1(1.1-3.5)$ & $2.0(1.2-2.9)$ & $4.0(I . I-7.5)$ & $1.4(1.2-1.8)$ & $0.1(0.0-0.2)$ & $0.1(0.0-0.3)$ \\
\hline Oxygen desaturation (mild, short) & 4 & $5.3(2.6-8.9)$ & $4.5(2.2-8.3)$ & $4.2(1.8-8.6)$ & $2.2(1.7-3.3)$ & $0.2(0.0-0.6)$ & $0.2(0.0-0.4)$ \\
\hline Hypertension & 5 & $3.3(1.5-5.2)$ & $1.9(1.0-2.8)$ & $2.4(1.1-3.8)$ & $\mathrm{I} .8(1.4-2.0)$ & $0.0(0.0-0.1)$ & $0.0(0.0-0.1)$ \\
\hline Apnea (not prolonged) & 6 & $6.2(2.6-9.9)$ & $5.8(2.2-12.3)$ & $6.5(1.6-11.4)$ & $1.6(1.1-2.2)$ & $0.3(0.1-0.7)$ & $0.1(0.0-0.1)$ \\
\hline Oxygen desaturation (mild, long) & 7 & $6.3(3.7-9.5)$ & $2.8(1.1-5.6)$ & $3.6(2.1-5.9)$ & $1.4(1.1-1.8)$ & $0.3(0.1-0.6)$ & $0.2(0.0-0.3)$ \\
\hline AW obstruction & 8 & $5.0(2.3-10.8)$ & $5.0(1.5-11.6)$ & $5.0(2.0-7.0)$ & $1.9(1.3-2.0)$ & $0.1(0.0-0.2)$ & $0.0(0.0-0.2)$ \\
\hline Failed sedation & 9 & $6.9(4.3-10.0)$ & $8.8(4.4-14.1)$ & $4.5(1.2-9.9)$ & $1.3(1.0-1.4)$ & $0.0(0.0-0.0)$ & $0.0(0.0-0.0)$ \\
\hline Apnea (long) & 10 & $6.5(3.8-12.5)$ & $5.1(2.2-8.3)$ & $4.7(2.3-14.3)$ & $\mathrm{I} .8(1.4-2.0)$ & $0.3(0.1-0.7)$ & $0.1(0.0-0.2)$ \\
\hline Allergy & 11 & $9.7(4.8-20.4)$ & $15.4(7.2-30.0)$ & $\mid 3.8(6.2-2 \mid .7)$ & $2.1(1.5-3.0)$ & $0.2(0.0-0.5)$ & $0.3(0.0-1.3)$ \\
\hline Oxygen desaturation (severe) & 12 & $19.1(9.5-27.4)$ & I $5.0(7.8-20.3)$ & $20.3(\mid 1.0-31.0)$ & $2.2(1.7-2.6)$ & $0.8(0.4-1.9)$ & $0.6(0.3-0.9)$ \\
\hline Cardiovascular collapse & 13 & $49.1(31.7-68.0)$ & $54.1(35.9-76.5)$ & $56.2(43.4-79.6)$ & $3.8(3.0-4.6)$ & $3.0(2.2-4.0)$ & $2.4(1.5-3.6)$ \\
\hline Cardiac arrest & 14 & $85.5(50.8-92.4)$ & $94.0(85.1-97.7)$ & $98.3(95.5-99.9)$ & $7.6(5.8-9.0)$ & $24.8(11.4-39.4)$ & I $4.0(8.1-25.5)$ \\
\hline Seizure & 15 & $47.6(25.6-75.6)$ & $71.2(40.9-96.4)$ & $69.3(44.7-84.1)$ & $2.4(2.0-2.9)$ & $1.8(0.0-8.0)$ & $0.2(0.0-1.1)$ \\
\hline
\end{tabular}

Notes: Median global weighted mean outcomes per AE as percentages or length of unplanned hospital stays in days. Values are derived from I0,000 bootstrap resampling replicates of reported survey data. Ranks range from most frequently (as found to be reported in this study) observed AEs (I) to least frequently observed AEs (I5). Values in the parentheses are the interquartile range (IQR).

Abbreviations: AE, adverse event; AW, airway.

countries and found consistency of experience with AEs in all settings to be universally associated with increased costs. Reported rates of AEs in our study were consistent with those reported in previous retrospective studies. ${ }^{12-14}$ In addition, responses to our surveys from providers with experience and responsibility for sedation administration suggest that treatment patterns for AEs are largely similar across the globe despite differences in medical practice, sedatives used, site of service, and geography. In turn, the results of our analyses validate growing global concerns for the clinical and economic burden of procedural sedation and anesthesia in patient care across the world.

Few studies have examined clinical and treatment practice regarding sedation, despite the fact that an understanding of intervention frequencies may be fundamental to estimating the economic burden and costs of sedation-related AEs. One recent 21-question survey of endoscopic procedure sedation comprised 33 survey responses across 16 European countries and concluded that there was a high degree of variability in practice and outcomes. ${ }^{21}$ In contrast, our study featured a higher sampling rate per country and found general similarity in sedation experiences and practices. In particular, both our own and other studies have determined similar trends in the use of available patient monitoring modalities, with providers reporting a relatively high reliance on pulse oximetry and blood pressure, with only moderate use of capnography among respondents reported. ${ }^{21}$ The global practice of relying on pulse oximetry to monitor for impending AEs may explain the consistent rate at which AEs are detected.
To our knowledge, there have been no previous determinations of comprehensive costs for interventions associated with sedation-related AEs that can serve as comparison for our results. A single study has reported a direct cost for tracheal intubation in the US of \$120 in 2009 (approximately $\$ 134$ adjusted to 2016 USD), ${ }^{22}$ consistent with the global mean cost of $\$ 133.81$ returned in our analysis. Another study of medical claims costs associated with sedation-related AEs used the US Premier database and yielded vastly different results from those of our study ${ }^{23}$ However, that analysis included legal fees and was disproportionately associated with significant morbidity and high downstream costs, while ours was focused on direct costs of health care resources. ${ }^{23}$ Nevertheless, it is an important reality that increased costs resulting from sedation-related AEs are driven by not only the costs of interventions used to treat them but also the outcome or longer-term impact of the complication.

Our study consistently found across countries that even relatively minor AEs, such as mild oxygen desaturation, added to the cost of care. For the subset of most common AEs reported, use of incidence data from other studies resulted in calculated cost increases of $13 \%$ and $23 \%$ above those of uncomplicated procedural sedations in Germany and the US, respectively. As these estimates use only a subset of all AEs, the overall impact of AEs on procedural sedation costs is likely higher. This is supported by payer expectation, whereby respondents in these countries indicated cost increases of $78 \%$ and $58 \%$, respectively. As calculated increases were based only on a subset of AEs for which incidence data 
were available, the true cost increase may be closer to payer expectations. Our estimate also excludes costs from very rare, severe AEs and the costs of their potential outcomes. Even for the subset of AEs for which incidence data were available, the increase in costs can be considerable when considering that $\sim 4$ million procedural (nonoperating room) sedations occurred in the US per year over the period from 2010 to $2014 .^{2}$ At this level, the subset of AEs would equate to a median additional burden of $\$ 2.2$ billion/year.

Clearly, an important contributor to costs is the frequency with which AEs are observed, which is directly related to medical practice. Sedation agents used, guidelines followed, and choice of monitoring modalities affect both AE frequency and treatment response. A common perception among providers from the authors' experience is that the occurrence of minor events is not seen as an important safety or quality indicator. The results presented here, however, demonstrate that substantial burden is placed on health care resources if these events are not prevented. Furthermore, what begin as minor events may herald the oncoming of more serious AEs, and medical practice, such as monitoring modality, may contribute to the early recognition of these events. Economic and provider benefits could therefore potentially be realized if greater importance was placed on prevention of even minor AEs, and their early recognition should constitute good clinical practice. ${ }^{18}$

An additional contributor to costs and potential explanation of individual- and country-level variation in the responses may lie in variations in interpretations of SIVA definitions of AEs and interventions. For example, short, mild oxygen desaturation is defined by SIVA as arterial oxygen saturation between $75 \%$ and $90 \%$ for $<60$ seconds, while non-prolonged apnea (not connected to AW obstruction) is defined as lasting up to 60 seconds. The difference in patient impact of 50 seconds of $75 \%$ oxygen saturation versus $90 \%$ oxygen saturation may be associated with vast differences in interventions, thereby resulting in vastly different costs. Additionally, variability in costs of different interventions across countries (eg, AW repositioning and tactile stimulation were found to range from $£ 0$ [UK] to $\$ 73$ [US] and €9 [France] to $\$ 325$ [US], respectively) may reflect differences in how interventions are locally defined. In general, costs were higher in the USA, which may reflect local care practices, such as more prevalent use of anesthesiology providers and use of monitored anesthesia care. The impact of concerns for risk management and litigation in the USA after AEs occur may also differ from that of other countries and affect costs. The current survey was not designed to be able to tease apart these potential confounding factors.
Our study design, including our decision to obtain pilot data, yielded both advantages and limitations. Our response targets were feasible and provided a sample of practice and costs across five countries. Our data were collected via a third-party intermediate, which allowed us to blind the authors to respondents and vice versa. This study presents the first estimate of both health care and cost burden specific to procedural sedation. As no specific costing methodology was enforced by the survey, it is possible that payers provided cost data using various methods, limiting comparability. On the other hand, we did not include items that could be interpreted to have a direct and additional opportunity cost (such as early termination of a procedure) in the presented direct cost calculations.

In examples where results of our study have coincided with published results, value agreements have been good, suggesting that our small sample size still yielded results consistent with larger, focused studies. Nevertheless, caution must be employed in how far our results may be interpreted. The pseudorandom sampling of AEs for estimated intervention and outcomes meant that some respondents answered questions on treatment practice for AEs that they may not have seen within the last year. Nevertheless, we feel it is reasonable to assume that given their clinical education and previous experience, respondents were able to provide sensible reports of their typical treatment patterns. The organization of the survey also did not allow for all questions to be directly linked, such that cause and effect between questions cannot be guaranteed. Given its undertaking as a pilot study, the countries included and the number of responses per country were limited with respect to other published surveys. With this pilot study completed, the authors are keen to extend the reach of this survey in collaboration with interested societies.

\section{Conclusion}

Clinical experiences with AEs during procedural sedation can be generalized internationally, and both minor and severe AEs should be recognized to result in poor patient outcomes (ie, substantial delays to or cancellation of planned procedures). Furthermore, all sedation-related AEs and by extension the interventions performed to maintain patient safety should be recognized to have associated costs. The findings reported here should be considered as a first estimate of event burden during procedural sedation. Extension of this survey to include more respondents and additional geographies is required to clarify the significance of the findings reported.

Our study found that clinical and treatment practices are in line with those expected from clinical experience. The cost 
of AEs, however, was found to be more than anticipated in certain cases, which may drive further discussion about the best ways to optimize patient safety to provide value-based health care. The findings of our analyses also suggest that even minor AEs can add considerable costs when considering the increasing number of procedural sedations performed. Costs of sedation-related AEs may be partially controlled by improvements in monitoring to reduce their occurrence and the need for interventions to treat them.

\section{Disclosure}

RS is the owner and JAD an employee of Coreva Scientific $\mathrm{GmbH} \& \mathrm{Co}$. KG, which received consultancy fees for performing, analyzing, and communicating the work presented here. RW is an employee of Medtronic Inc., which funded the research. JRL and DKW did not receive remuneration from Medtronic Inc. PK received an honorarium from Medtronic Inc. JRL is a contracted medical consultant for Coreva Scientific $\mathrm{GmbH} \& \mathrm{Co}$. KG. The authors report no other conflicts of interest in this work.

\section{References}

1. Adams MA, Saleh A, Rubenstein JH. A systematic review of factors associated with utilization of monitored anesthesia care for gastrointestinal endoscopy. Gastroenterol Hepatol (N Y). 2016;12(6):361-370.

2. Nagrebetsky A, Gabriel RA, Dutton RP, Urman RD. Growth of nonoperating room anesthesia care in the United States: a contemporary trends analysis. Anesth Analg. 2017;124(4):1261-1267.

3. Tetzlaff JE, Vargo JJ, Maurer W. Nonoperating room anesthesia for the gastrointestinal endoscopy suite. Anesthesiol Clin. 2014;32(2): 387-394.

4. Jin EH, Hong KS, Lee Y, et al. How to improve patient satisfaction during midazolam sedation for gastrointestinal endoscopy? World $J$ Gastroenterol. 2017;23(6):1098-1105.

5. The American Society of Anesthesiologists. Standards for Basic Anesthetic Monitoring. Vol. 2017. 2015. Anesthesia Monitoring Recommendations.

6. European Board of Anaesthesiology. European Board of Anaesthesiology (EBA) Recommendations for Minimal Monitoring during Anaesthesia and Recovery. Vol. 2017. 2016. EBA Recommendations for Monitoring during Anaesthesia.

7. Agostoni M, Fanti L, Gemma M, Pasculli N, Beretta L, Testoni PA. Adverse events during monitored anesthesia care for GI endoscopy: an 8-year experience. Gastrointest Endosc. 2011;74(2):266-275.

8. Beitz A, Riphaus A, Meining A, et al. Capnographic monitoring reduces the incidence of arterial oxygen desaturation and hypoxemia during propofol sedation for colonoscopy: a randomized, controlled study (ColoCap study). Am J Gastroenterol. 2012;107(8):1205-1212.
9. Klare P, Reiter J, Meining A, et al. Capnographic monitoring of midazolam and propofol sedation during ERCP: a randomized controlled study (EndoBreath study). Endoscopy. 2016;48(1):42-50.

10. Lightdale JR, Goldmann DA, Feldman HA, Newburg AR, DiNardo JA, Fox VL. Microstream capnography improves patient monitoring during moderate sedation: a randomized, controlled trial. Pediatrics. 2006; 117(6):e1170-e1178.

11. Qadeer MA, Vargo JJ, Dumot JA, et al. Capnographic monitoring of respiratory activity improves safety of sedation for endoscopic cholangiopancreatography and ultrasonography. Gastroenterology. 2009; 136(5):1568-1576; quiz 1819-1820.

12. Bellolio MF, Gilani WI, Barrionuevo P, et al. Incidence of adverse events in adults undergoing procedural sedation in the emergency department: a systematic review and meta-analysis. Acad Emerg Med. 2016;23(2):119-134.

13. McGrane O, Hopkins G, Nielson A, Kang C. Procedural sedation with propofol: a retrospective review of the experiences of an emergency medicine residency program 2005 to 2010. Am J Emerg Med. 2012;30(5):706-711.

14. Tohda G, Higashi S, Wakahara S, Morikawa M, Sakumoto H, Kane T. Propofol sedation during endoscopic procedures: safe and effective administration by registered nurses supervised by endoscopists. Endoscopy. 2006;38(4):360-367.

15. Jalili M, Bahreini M, Doosti-Irani A, Masoomi R, Arbab M, Mirfazaelian H. Ketamine-propofol combination (ketofol) vs propofol for procedural sedation and analgesia: systematic review and metaanalysis. Am J Emerg Med. 2016;34(3):558-569.

16. Bellolio MF, Puls HA, Anderson JL, et al. Incidence of adverse events in paediatric procedural sedation in the emergency department: a systematic review and meta-analysis. BMJ Open. 2016;6(6):e011384.

17. Poh YN, Poh PF, Buang SN, Lee JH. Sedation guidelines, protocols, and algorithms in PICUs: a systematic review. Pediatr Crit Care Med. 2014;15(9):885-892.

18. Mason KP, Green SM, Piacevoli Q; International Sedation Task Force. Adverse event reporting tool to standardize the reporting and tracking of adverse events during procedural sedation: a consensus document from the World SIVA International Sedation Task Force. Br J Anaesth 2012;108(1):13-20.

19. Friedrich-Rust M, Welte M, Welte $C$, et al. Capnographic monitoring of propofol-based sedation during colonoscopy. Endoscopy. 2014;46(3): 236-244.

20. Mehta PP, Kochhar G, Albeldawi M, et al. Capnographic monitoring in routine EGD and colonoscopy with moderate sedation: a prospective, randomized, controlled trial. Am J Gastroenterol. 2016;111(3): 395-404.

21. Vaessen HH, Knape JT. Considerable variability of procedural sedation and analgesia practices for gastrointestinal endoscopic procedures in Europe. Clin Endosc. 2016;49(1):47-55.

22. Gupta D, Wang H. Cost-effectiveness analysis of flexible optical scopes for tracheal intubation: a descriptive comparative study of reusable and single-use scopes. J Clin Anesth. 2011;23(8):632-635.

23. Saunders R, Erslon M, Vargo J. Modeling the costs and benefits of capnography monitoring during procedural sedation for gastrointestinal endoscopy. Endosc Int Open. 2016;4(3):E340-E351.

\section{Dovepress}

\section{Publish your work in this journal}

Therapeutics and Clinical Risk Management is an international, peerreviewed journal of clinical therapeutics and risk management, focusing on concise rapid reporting of clinical studies in all therapeutic areas, outcomes, safety, and programs for the effective, safe, and sustained use of medicines. This journal is indexed on PubMed Central, CAS,
EMBase, Scopus and the Elsevier Bibliographic databases. The manuscript management system is completely online and includes a very quick and fair peer-review system, which is all easy to use. Visit $\mathrm{http}: / / \mathrm{www}$.dovepress.com/testimonials.php to read real quotes from published authors. 